\title{
Spin-Dependent Enantioselective Electropolymerization
}

\author{
Francesco Tassinari,* Dana Amsallem, Brian P. Bloom, Yiyang Lu, Anjan Bedi, David H. Waldeck, \\ Ori Gidron, and Ron Naaman
}

Cite This: https://dx.doi.org/10.1021/acs.jpcc.0c06238

Read Online

ABSTRACT: The electro-oxidative polymerization of an enantiopure chiral 3,4-ethylenedioxythiophene monomer, performed using spin-polarized currents, is shown to depend on the electron spin orientation. The spin-polarized current is shown to influence the initial nucleation rate of the polymerization reaction. This observation is rationalized in the framework of the chiral-induced spin selectivity effect.

\section{INTRODUCTION}

The investigation of electrochemical reactions under an applied magnetic field, magnetoelectrochemistry, has been growing over the past few decades. It is generally accepted that an external magnetic field can be used to affect kinetic parameters; ${ }^{1,2}$ however, magnetic fields are not believed to provide an enantioselectivity for electrochemical reactions of chiral molecules. ${ }^{3}$ The recent discovery of the chiral-induced spin selectivity (CISS) effect, ${ }^{4}$ which describes the enantiospecific interaction of chiral molecules and an electron's spin, is refining our understanding of magnetic effects by revealing how magnetism might be used to introduce dissymmetry into electrochemical reactions. CISS-based studies show how an external magnetic field can be used to generate spin-polarized electrons from a ferromagnetic electrode, which are then used to induce a chiral bias into redox reactions. This approach has been applied to influence the efficiency of water-splitting reactions by manipulating the relative importance of singlet and triplet reaction pathways ${ }^{5,6}$ and is also the basis for a growing body of work called spin-dependent electrochemistry, ${ }^{7-10}$ where the spin-polarization is used to govern enantioselective interactions. This concept was recently demonstrated for the electropolymerization of achiral monomers to form chiral polymer films by controlling the spinpolarization of the working electrode; ${ }^{11}$ however, the underlying mechanism is still unclear.

In this study, the electropolymerization of chiral-modified EDOT monomers, [(2R,3R)-2,3-diphenyl-3,4-ethylenedioxythiophene $(R, R$-EDOT) and $[(2 S, 3 S)$-2,3-diphenyl-3,4-ethylenedioxythiophene] ( $S, S$-EDOT), was investigated by using ferromagnetic substrates. Chiral poly-EDOT (PEDOT) has been previously studied as an enantioselective electrode for

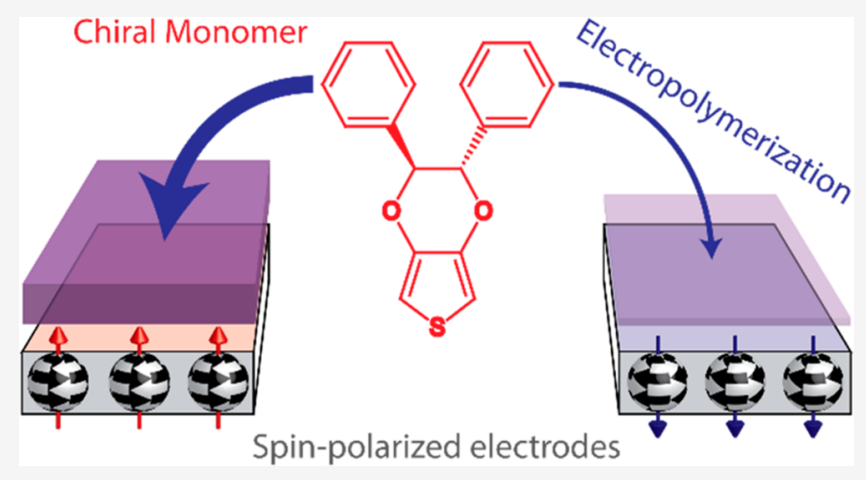

analytical and electrochromic applications. ${ }^{12-14}$ The present work examines the kinetics for the electrochemical growth of chiral PEDOT films from chiral monomer units and its dependence on the spin polarization in the ferromagnetic working electrode.

\section{METHODS}

Synthesis and Purification of the EDOT Monomer. 3,4-Dimethoxythiophene $(0.54 \mathrm{~mL}, 4.5 \mathrm{mmol}),(R, R)$ (+)-hydrobenzoin (9 mmol, $1.93 \mathrm{~g}), p$-toluenesulfonic acid ( $86 \mathrm{mg}, 0.45 \mathrm{mmol})$, and toluene $(12 \mathrm{~mL})$ were added to a two-necked flask under a nitrogen purge. The flask was heated at $90{ }^{\circ} \mathrm{C}$ for $72 \mathrm{~h}$ and then cooled to room temperature. The solvent was evaporated, and the dark oil was purified by flash chromatography $\left(\mathrm{SiO}_{2}\right.$, hexane) to give $R, R$-EDOT (180 mg, $30 \%)$ as a white solid. S,S-EDOT was prepared using the same procedure with yields of $30 \% .{ }^{1} \mathrm{H} \mathrm{NMR}\left(500 \mathrm{MHz}, \mathrm{CDCl}_{3}\right): \delta$ 6.48 (s, 2H H-C(1)), 4.96 (s, 2H, H-C(3)), 7.04-7.06 (m, $2 \mathrm{H}, \mathrm{H}-\mathrm{C}(5)), 7.19-7.24(\mathrm{~m}, 6 \mathrm{H}, \mathrm{H}-\mathrm{C}(6), \mathrm{H}-\mathrm{C}(7)) .{ }^{13} \mathrm{C}$ NMR (126 MHz, $\left.\mathrm{CDCl}_{3}\right): \delta 99.8(\mathrm{C} 1), 142.39(\mathrm{C} 2), 81.23$ (C3), 135.75 (C4), 127.61 (C5), 128.25 (C6), 128.69 (C7). HR-LC-MS $m / z$ (\%): 294.0715 (100, [M]). Calcd. for $\mathrm{C}_{18} \mathrm{H}_{14} \mathrm{O}_{2} \mathrm{~S}: 294.0714$. UV-vis (acetonitrile): $\lambda_{\max }(\varepsilon)=258$ nm.

Received: July 8, 2020

Revised: August 19, 2020

Published: August 31, 2020 
Electropolymerization Experiments. A semitransparent working electrode, prepared by electron beam evaporation on a glass slide of a thin nickel layer capped with a thin gold layer (glass/5 nm Ti/15 nm Ni/5 nm Au), was used for the electropolymerization. The electrochemical cell was a single compartment cell, equipped with a silver wire as a pseudo reference electrode and a platinum wire as the counter electrode. The monomer was dissolved in anhydrous acetonitrile to give a $6 \mathrm{mM}$ solution, with $50 \mathrm{mM}$ tetrabutylammonium-tetrafluoborate (TBATFB) as the supporting electrolyte.

The electropolymerization was performed under chronoamperometric conditions, with a potential step of $1.5 \mathrm{~V}$. No preconditioning steps were used. The polymerization proceeded until a total charge of $10 \mathrm{mC}$ was reached, and then, a reducing step of $-1 \mathrm{~V}$ was applied to the obtained polymer film for $20 \mathrm{~s}$ to neutralize the doping. During the electropolymerization, an external magnetic field of $\sim 0.4 \mathrm{~T}$ was applied to the nickel electrode, by placing a strong neodymium permanent magnet on the back of the working electrode. The applied field is sufficient to magnetize the nickel film out-ofplane. The resulting chiral PEDOT-functionalized electrodes were then rinsed with acetonitrile and dried gently with a nitrogen flow before characterization.

Electrochemical Quartz Microbalance Experiment. Custom quartz crystals coated with a $100 \mathrm{~nm}$ layer of $\mathrm{Ni}$ followed by $10 \mathrm{~nm}$ of $\mathrm{Au}$, to prevent oxidation, were purchased from $\mathrm{CH}$ Instruments. The fundamental frequency of the crystals was $7.995 \mathrm{MHz}$. A platinum wire was used as the counter electrode, and a silver wire was used as a pseudoreference electrode. For the electopolymerization, a $0.25 \mathrm{mg}$. $\mathrm{mL}^{-1}$ solution of $(\mathrm{S}, \mathrm{S})$ - or $(\mathrm{R}, \mathrm{R})$-EDOT monomer was dissolved in acetonitrile with $0.1 \mathrm{M}$ tetrabutylammonium perchlorate as the supporting electrolyte. The electropolymerization was performed by applying a potential of $1.5 \mathrm{~V}$ for 10 $\mathrm{s}$, followed by a potential of $-0.5 \mathrm{~V}$ for an additional $10 \mathrm{~s}$. During electropolymerization, an external magnet was positioned under the electrochemical cell, resulting in a $0.3 \mathrm{~T}$ magnetic field strength on the surface of the quartz crystal.

\section{RESULTS AND DISCUSSION}

Figure 1 shows typical chronoamperometric curves for the electropolymerization of $R, R$-EDOT and $S, S$-EDOT monomers on a ferromagnetic electrode, while the electrode is magnetized either north (red curves) or south (blue curves) by an external magnetic field. The magnetization is considered to be north when the lines of the magnetic field are going into the electrode surface from the electrolyte solution and south when the field lines are going out of the electrode surface into the electrolyte solution. The current density versus time plots can be described as a three phase process, which follows the generally accepted mechanism for the electropolymerization process. ${ }^{15}$ First, the current decreases because of the double layer formation, and this appears to happen in conjunction with the initial oxidation of monomers near the surface. As the concentration of the oxidized monomer increases, radical coupling begins to occur and oligomers form close to the electrode surface. Once the oligomers reach a critical length, they become insoluble, deposit on the surface of the electrode, and act as nucleation points for the continued growth of the polymer chains on the surface. The second phase is characterized by a current increase, with the slope of the
A

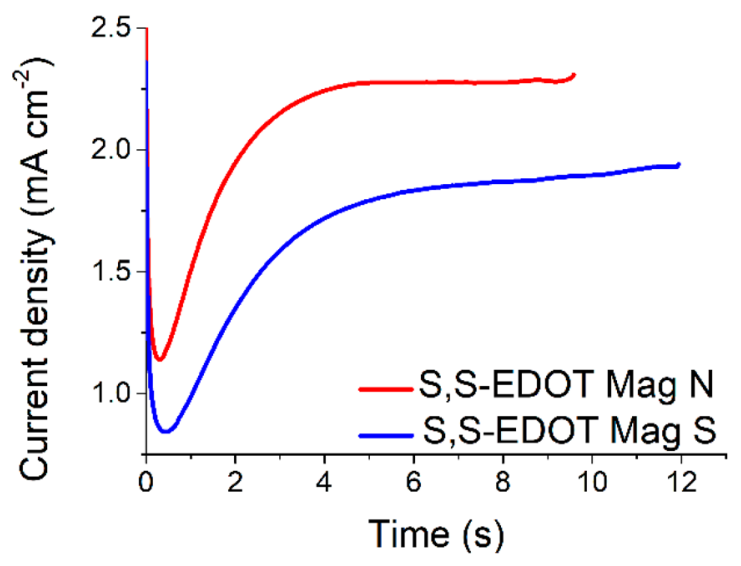

B

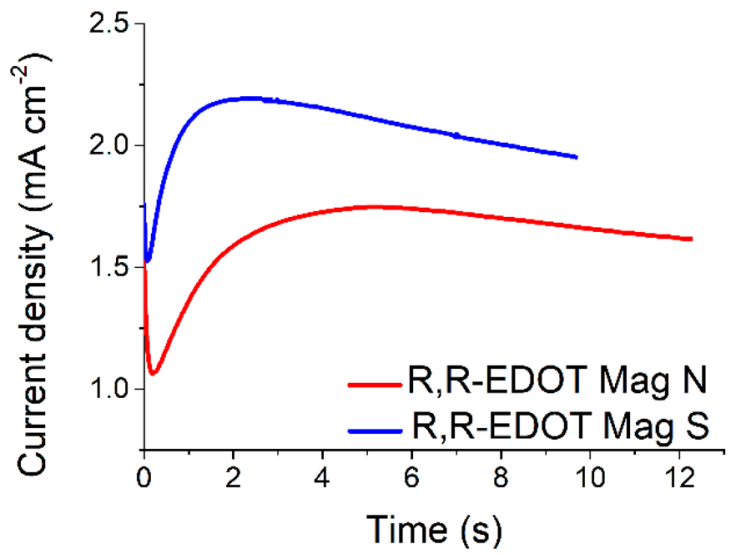

Figure 1. Chronoamperometric studies of the electropolymerization of (A) S,S-EDOT and (B) R,R-EDOT using a ferromagnetic working electrode under the application of a north (red) and south (blue) magnetic field. The polymerization was stopped after a target charge of $10 \mathrm{mC}$ was reached (6 mM EDOT concentration, anhydrous ACN with $50 \mathrm{mM}$ TBATFB as the supporting electrolyte, $1.5 \mathrm{~V}$ vs Ag wire). The magnetic field strength at the surface of the electrode is $\sim 0.45 \mathrm{~T}$.

current being proportional to the reaction rate of the electropolymerization under diffusion control. In the third phase, the current reaches a plateau, indicating that the polymerization rate in this stage is limited by the charge transfer rate through the film rather than monomer diffusion. Figure 1 shows that the handedness of the monomer and the orientation of the magnet used to magnetize the electrode determine the slope in the second phase of the electropolymerization. For instance, the $R, R$-EDOT monomer exhibits a much sharper slope with north magnetization than for south magnetization. Conversely, the opposite dependence is observed for the $S, S$-EDOT monomer. Thus, the growth kinetics are affected by the magnetic field direction.

To characterize the product, circular dichroism (CD) and absorption spectra of the polymer films prepared under north (red) and south (blue) magnetization were measured and compared with films produced with no magnetic field applied during electropolymerization (black), as shown in Figure 2A and $2 \mathrm{~B}$ (note that the optical measurements were performed on the reduced films). In the absorption spectra, a main peak at $600 \mathrm{~nm}$ with a shoulder at $560 \mathrm{~nm}$ and a smaller peak at 655 $\mathrm{nm}$ are visible. In the $\mathrm{CD}$ spectra the two main peaks are clearly visible at the same wavelengths as in the UV-vis 
A
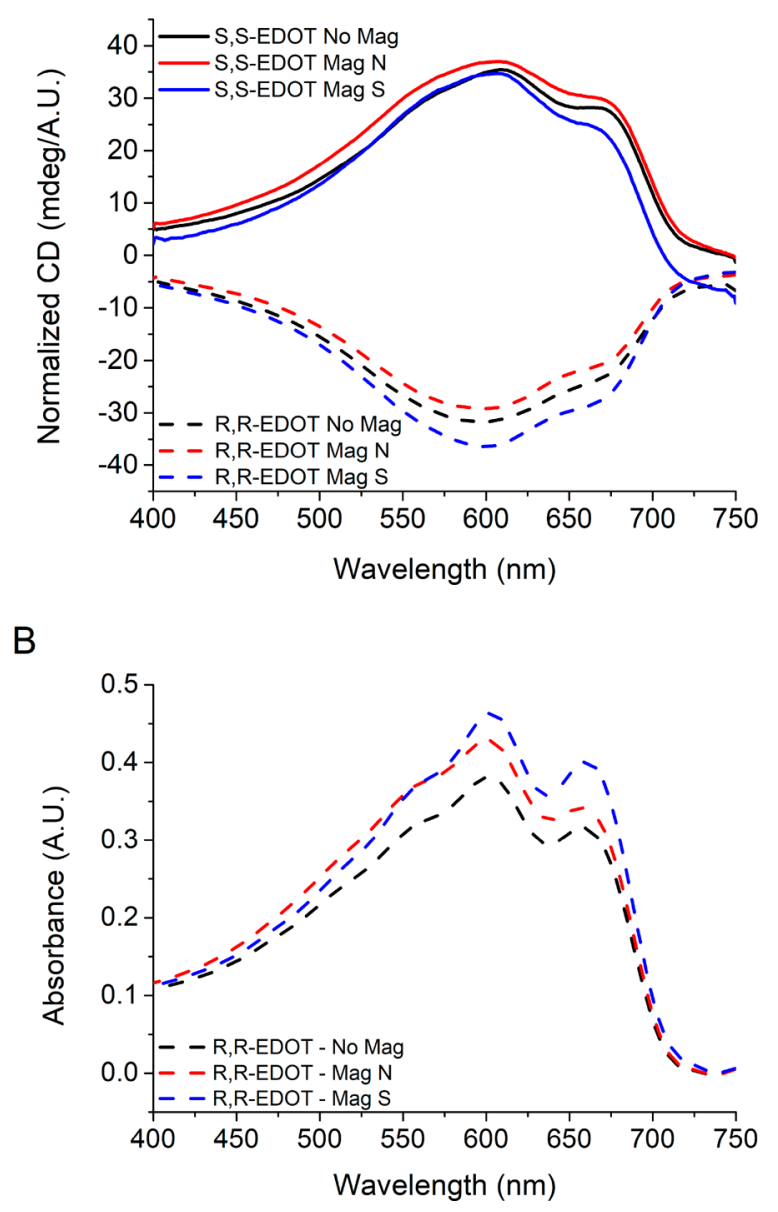

Figure 2. (A) Normalized circular dichroism spectra of the $S, S$ PEDOT (solid line) and R,R-PEDOT (dashed line) films electropolymerized under a magnetic field, north (red) and south (blue), and without a magnetic field (black). (B) UV-vis spectra of the $R, R$ PEDOT electropolymerized films (S,S-EDOT spectra in the Supporting Information (SI)). All the films have been electrodeposited by a total charge of $10 \mathrm{mC}$.

spectra. We interpret the difference in the intensity of the absorption spectra of the polymerized films as an indication of different thicknesses. These data are corroborated by profilometry measurements; see Table 1 for polymer thickness.

Table 1. Profilometry Measurements of R,R-EDOT and $S, S$ EDOT Electropolymerized Films with and without the Application of an External Magnetic Field

\begin{tabular}{lccc} 
& no magnet & magnetic north & magnetic south \\
$R, R$-EDOT & $79 \pm 5 \mathrm{~nm}$ & $80 \pm 5 \mathrm{~nm}$ & $116 \pm 5 \mathrm{~nm}$ \\
$S, S$-EDOT & $63 \pm 5 \mathrm{~nm}$ & $120 \pm 5 \mathrm{~nm}$ & $90 \pm 5 \mathrm{~nm}$ \\
\hline
\end{tabular}

The data show that the polymer films are thicker if a magnetic field is applied during electropolymerization, despite the same amount of charge being passed in the electrodeposition. The effect of the electropolymerization thickness with an external magnetic field is consistent with literature reports. ${ }^{16}$ Interestingly, $R, R$-PEDOT films polymerized under south magnetization are thicker than films prepared under north magnetization. Conversely, the opposite trend holds for S,S-PEDOT; films electropolymerized with north magnetization are thicker than south magnetization. Thus, a clear difference in the thickness of the polymer film for north and south magnetization occurs, and this difference is enantiospecific, despite the same amount of charge being passed during electropolymerization.

The circular dichroism (CD) spectra of the films, normalized by their maximum absorbance value at $655 \mathrm{~nm}$ to account for different film thicknesses, are reported in Figure 2A.

These findings show that the electropolymerization reaction yield depends on both the applied magnetic field direction and the handedness of the monomeric unit, i.e., the polymeric film thickness at fixed charge changes with the magnet direction and the monomer's enantiomeric form. Typically, the magnetic field effects on electrochemical reaction kinetics are attributed to either the Lorentz force ${ }^{1}$ or the Kelvin force ${ }^{2}$ that act on reactants and reaction intermediates. The Lorentz force arises from magneto-hydrodynamic effects under a current flow, whereas the Kelvin force arises from magnetic field gradients acting on paramagnetic species, such as organic radicals formed during the monomer electro-oxidation. These forces can influence the mass transfer of the monomer close to the electrode surface and cause an increase in the polymerization rate for low concentrations of the monomer (below $0.2 \mathrm{M}){ }^{17}$ Neither of these effects should be sensitive to the sign of the applied magnetic field; however, the enantiospecificity with the sign (parallel or antiparallel to the surface normal) of the field is unprecedented.

To better elucidate the kinetic differences, experiments were performed using an electrochemical quartz crystal microbalance (EQCM). In this experiment, the current still indicates the amount of monomer oxidized per unit of time; however, the change in the frequency of the quartz crystal with time provides the real-time growth of the polymer film. Figure 3 shows EQCM data for quartz crystals coated with ferromagnetic electrodes, comprising $100 \mathrm{~nm}$ of $\mathrm{Ni}$ with a 10 $\mathrm{nm} \mathrm{Au}$ overlayer. The change in frequency and the corresponding mass change are presented for $R, R$-EDOT (up) and $S, S$-EDOT (down) monomers electropolymerized with a magnetic field applied in the north (red) and south (blue) orientation. The change in mass with the enantiomer and magnetic field direction is consistent with the thickness measurements reported vide supra.

An efficiency for the polymerization was calculated by comparing the integrated current to the change in mass. If every electron contributes to the growth of the polymer and 2.25 electrons are required for the growth of the polymer chain by one monomeric unit (the addition of one monomer to the chain requires two electrons, and the PEDOT chain in its doped state has one charge for every four units) then the total charge can be used to indicate the theoretical amount of polymer formed during the reaction. This change in mass was then compared with the change in mass measured by the quartz crystal microbalance during the electropolymerization to calculate a reaction conversion efficiency (Table 2). Clearly, the efficiency depends on the magnetic field orientation and on the handedness of the monomer. Assuming that the current is only associated with monomer oxidation, these data prove that when the magnet is in a "favorable" configuration the probability of polymer growth is higher.

Next, the effect of the magnetic field orientation was used to investigate the electropolymerization rate. The time-dependent 


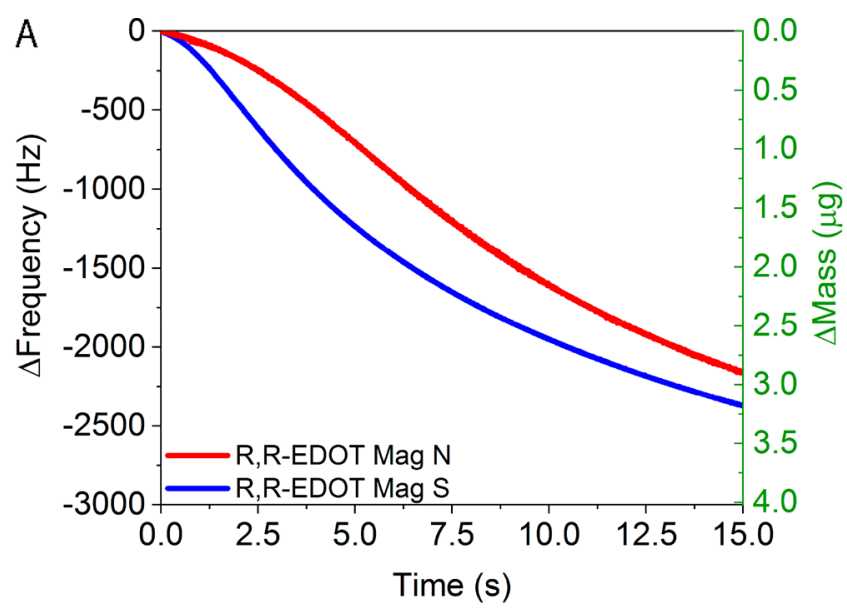

B

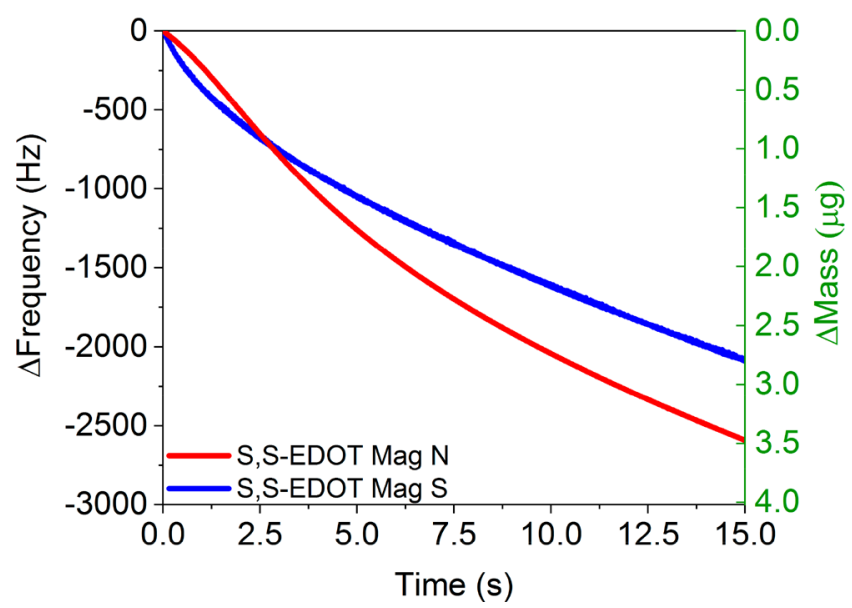

Figure 3. Quartz crystal microbalance results for the electropolymerization of (A) $R, R$-EDOT and (B) S,S-EDOT performed under different magnetic fields at a monomer concentration of 0.85 $\mathrm{mM}$.

Table 2. Reaction Efficiencies Derived from the QCM Experiments $^{a}$

\begin{tabular}{|c|c|c|c|c|}
\hline & \multicolumn{2}{|c|}{$R, R$-EDOT } & \multicolumn{2}{|c|}{$S, S$-EDOT } \\
\hline & $\begin{array}{l}\text { magnetic } \\
\text { north }\end{array}$ & $\begin{array}{l}\text { magnetic } \\
\text { south }\end{array}$ & $\begin{array}{l}\text { magnetic } \\
\text { north }\end{array}$ & $\begin{array}{l}\text { magnetic } \\
\text { south }\end{array}$ \\
\hline total charge $(\mathrm{mC})$ & 2.8 & 2.6 & 2.9 & 2.6 \\
\hline $\begin{array}{l}\text { theoretical mass } \\
(\mu \mathrm{g})\end{array}$ & 3.8 & 3.5 & 4.0 & 3.5 \\
\hline $\begin{array}{l}\text { measured mass } \\
(\mu \mathrm{g})\end{array}$ & 2.9 & 3.2 & 3.5 & 2.8 \\
\hline efficiency (\%) & 77 & 91 & 88 & 79 \\
\hline
\end{tabular}

${ }^{a}$ The total charge is calculated by integration of the chronoamperometric curves (these data are reported in the Supporting Information).

change in the current for an electrochemical reaction can be expressed as

$$
i=\left(\frac{n F A D_{0}^{1 / 2} C_{0}}{t^{1 / 2}}\right) \lambda \mathrm{e}^{\lambda^{2}} \operatorname{erfc}(\lambda)
$$

where $n$ is the number of electrons for the electrochemical process, $F$ is Faraday's constant, $D_{0}$ is the diffusion coefficient of the monomer, $C_{0}$ is the bulk concentration of the monomer, $t$ is time, and $\lambda$ is equal to $k_{\mathrm{f}} \sqrt{ } t / D_{0}$ in which $k_{\mathrm{f}}$ is the polymerization rate constant. Assuming that the change in mass measured by the QCM is proportional to the total charge during electropolymerization, namely

$$
\Delta m \propto \int_{t_{0}}^{t} i(t) \mathrm{d} t
$$

we are able to fit the change in mass over time measured by the QCM to find the polymerization rate (details of this procedure are described in the SI). In addition to $k_{\mathrm{f}}$, the fitting uses a scaling prefactor $Z$ and a time shift $x^{\prime}$ to account for the induction time of the polymerization reaction. The parameters in Tables 3 and 4 display a mirror symmetry for the effective

Table 3. Fit Parameters of the QCM Experimental Data for a Monomer Concentration of $0.85 \mathrm{mM}$

\begin{tabular}{lccc} 
& $Z$ & $x^{\prime}$ & $k_{\mathrm{f}}(\mathrm{cm} / \mathrm{s})$ \\
$\begin{array}{l}\text { magnetic north } \\
R, R \text {-EDOT }\end{array}$ & 11.3 & 1.5 & $7.28 \times 10^{-2}$ \\
$\begin{array}{l}\text { magnetic south } \\
R, R \text {-EDOT }\end{array}$ & 15 & 1.8 & $8.25 \times 10^{-2}$ \\
$\begin{array}{l}\text { magnetic north } \\
S, S \text {-EDOT }\end{array}$ & 16.2 & 1.9 & $8.37 \times 10^{-2}$ \\
$\begin{array}{l}\text { magnetic south } \\
S, S \text {-EDOT }\end{array}$ & 11 & 1.4 & $7.42 \times 10^{-2}$ \\
\hline
\end{tabular}

Table 4. Fit Parameters of the QCM Experimental Data for

\begin{tabular}{|c|c|c|c|}
\hline & $Z$ & $x^{\prime}$ & $k_{\mathrm{f}}(\mathrm{cm} / \mathrm{s})$ \\
\hline $\begin{array}{l}\text { magnetic north } \\
R, R \text {-EDOT }\end{array}$ & 23 & 0.6 & $6.93 \times 10^{-2}$ \\
\hline $\begin{array}{l}\text { magnetic south } \\
R, R \text {-EDOT }\end{array}$ & 30 & 0.4 & $8.49 \times 10^{-2}$ \\
\hline
\end{tabular}
a Monomer Concentration of $1.7 \mathrm{mM}$

rate constant $k_{\mathrm{f}}$, the characteristic induction time for film growth $x^{\prime}$, and the overall scaling factor $Z$ with respect to the monomer handedness and magnetic field. When the reactant concentration was increased from $0.85 \mathrm{mM}$ (Table 3) to 1.7 $\mathrm{mM}$ (Table 4) the scaling factor, which includes a concentration term, doubles. The induction time of the reaction also decreases, which indicates that the reaction initiates faster with a higher monomer concentration.

To probe the dependence of the polymerization reaction rate constant on the magnetization of the electrode, we also performed chronoamperometric polymerization experiments at different monomer concentrations. Below we describe a twostep model for the polymer growth that has been successfully applied to the electropolymerization of conductive polymers. ${ }^{18}$ In this model, the current takes the form given in eq 3 :

$$
i=a \sqrt{t}\left[1-\exp \left(-b t^{2}\right)\right]+c\left[1-\exp \left(-d t^{2}\right)\right]
$$

The first term of the equation describes the nucleation process and polymer growth under diffusion control, while the second term corresponds to an instantaneous nucleation process and growth under charge transfer control. The parameters $a, b, c$, and $d$ are given as

$$
a=\frac{n F A C \sqrt{D}}{\sqrt{\pi}}
$$




$$
\begin{aligned}
& b=\frac{2 k A N_{\mathrm{diff}} \pi D}{3}\left(\frac{8 \pi C M}{\rho}\right)^{1 / 2} \\
& c=n F A k_{3 \mathrm{D}}^{\prime} \\
& d=\frac{\pi M^{2} A N_{3 \mathrm{D}} k_{3 \mathrm{D}}^{2}}{\rho^{2}}
\end{aligned}
$$

where $n$ is the number of electrons, $F$ is Faraday's constant, $A$ is the electrode's area, $D$ is the diffusion coefficient, $C$ is the monomer concentration, $k$ is the rate constant of nuclei formation, $N_{\text {diff }}$ is the number of nuclei formed at $t=0$ under diffusion control, $M$ is the molar mass of the monomer, $\rho$ is the density of the monomer, $k_{3 \mathrm{D}}$ and $k_{3 \mathrm{D}}^{\prime}$ are the rate constants of the 3D nuclei growth parallel and perpendicular to the surface, respectively, and $N_{3 D}$ is the number of instantaneous nuclei formed at $t=0$.

In the early stages of polymerization, the main contribution to the current density is dominated by diffusion, and hence, the second term in the equation can be neglected. This approximation is supported by the fact that the effect of the magnetic field is not observed at long polymerization times ( 5 min), for which the thickness of the polymeric film is mainly due to processes happening under charge transfer control. Moreover, it is expected that the spin polarization of the electrons transmitted through the chiral electropolymerized polymer has a typical length and the polarization decays with the distance. Thus, as the polymer film becomes thicker, the spin selectivity is expected to decrease, further corroborating that the nucleation step is largely affecting the resulting films. Fitting the amperometric curves to eq 3 gives the parameter $k$. $N_{\text {diff, }}$ which contains both the rate constant for the nuclei formation and the number of nuclei formed at early times. While in principle it is possible to determine $N_{\text {diff }}$ by the position of the amperometric peak, ${ }^{19}$ corrosion of the nickel substrate at long polymerization times can occur, which would affect the value for $N_{\text {diff }}$ Thus, a comparison of $k \cdot N_{\text {diff }}$ under different magnetizations is used instead. Figure 4 shows a plot of $k \cdot N_{\text {diff }}$ for $S, S$-EDOT under north (red) and south (blue) magnetizations. The difference in $k \cdot N_{\text {diff }}$ indicates that the kinetics for the electropolymerization at early time are sensitive

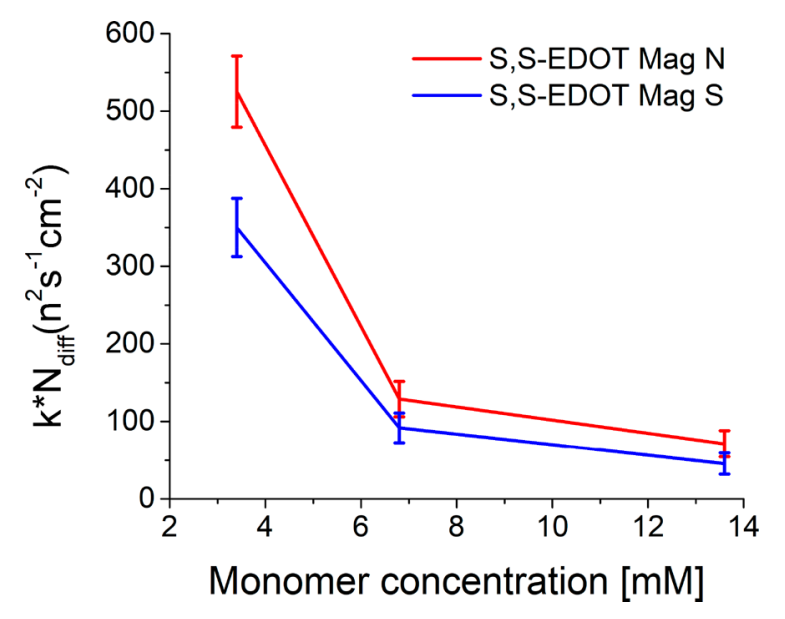

Figure 4. Graph of the calculated $k \cdot N_{\text {diff }}$ parameter versus the monomer concentration for $S, S$-EDOT polymerization under magnetic fields, north and south. The lines are a guide for the eye. to both the orientation of the magnetic field and the handedness of the monomeric unit.

The decrease of the $k \cdot N_{\text {diff }}$ parameter with the increase of monomer concentration is consistent with experiments for the electrochemical deposition of metals; ${ }^{19}$ the number of nuclei formed in the initial nucleation step decreases with the increase of the concentration of metal ions in solution. These data demonstrate that the nucleation step is influenced by the magnetization of the working electrode and can explain the thickness differences seen in the films polymerized under different magnetic fields.

The dependence of the polymer growth on the handedness of the monomer and on the magnetization direction of the working electrode can be explained by the chiral-induced spin selectivity (CISS) effect, i.e., a chiral molecule's interaction with an electron depends on the electron's spin direction and the handedness of the chiral molecule. When a molecule is in close proximity to the electrode's surface, a charge redistribution occurs in the molecule and an induced dipole is formed. For chiral molecules, this charge redistribution is accompanied by spin-polarization, and the direction of the spin depends on the handedness of the molecule. ${ }^{20} \mathrm{~A}$ similar effect has been attributed to the difference in the propensity for the adsorption of chiral molecules on magnetized surfaces in other experimental works. ${ }^{21,22}$ At the same time, the ferromagnetic electrode, under an external magnetic field, is spin-polarized due to the splitting of the spin population at the Fermi level. In this study, we rationalize the results using a model in which the spin on the monomer's radical intermediate at the electrode interacts with the spins present in the magnetized metal via exchange interactions. ${ }^{23}$ By exchange interactions we refer to the quantum effect for which the energy of two interacting electrons depends on the relative orientation of their spins. At the electrode interface, the wave functions of the monomer and of the magnetized surface overlap, forming a spatial hybrid wave function. This wave function can be either symmetric or antisymmetric, depending on the electrons' relative spin orientation, and can have two different electrostatic energies. Whether the interaction is favorable (spins aligned antiparallel, singlet-like) or unfavorable (spins aligned parallel, triplet-like) depends on the handedness of the molecule and the magnetization of the substrate. Scheme 1 summarizes the interaction differences between $R, R$-EDOT and $S, S$-EDOT with a north (top) and south (bottom) magnetized substrates. Effects on chemical reactions due to exchange interactions are known in photochemical experiments. The role of what is called spin catalysis, where the reactivity of a radical pair is enhanced by an exchange interaction with a paramagnetic species, is well documented in the literature. ${ }^{24}$ Conversely, the electropolymerization reaction studied in this work does not fall into this category, since the dependence of the effect on the direction of the magnetic field is unique to chiral systems. A chirality-dependent exchange interaction between the monomer and the spin-polarized surface is thus used to explain the symmetry breaking that is observed with the reversal of the magnetic field. It is important to note that other types of magnetic effects are invariant with the magnetic field orientation.

This enantiospecific interaction manifests in two important ways. First, the effective barrier height for electron injection depends on the spin alignment of the system, and this directly affects the reaction rate for the elementary electron transfer step. This rate impacts the polymerization rate constant $k_{\mathrm{f}}$, as 
Scheme 1. Graphical Description of the Interaction Model between the Magnetized Electrode and the Chiral Monomer

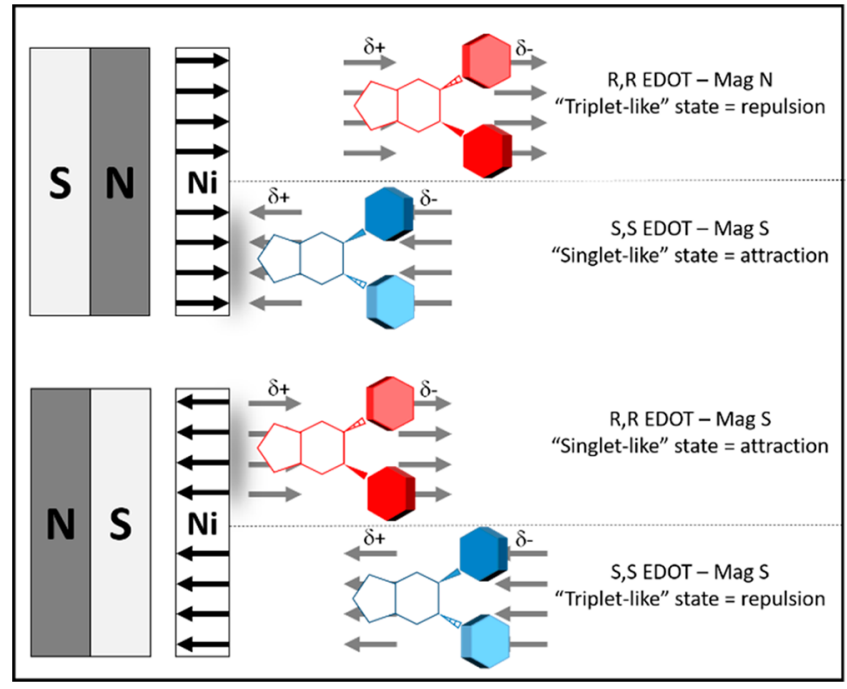

shown by the QCM data and its dependence on the magnetic field direction for the chiral monomers. Second, the magnetic field-dependent adsorption rate of the initial chiral oligomers onto the electrode surface could affect the nucleation rate of the reaction and hence the thickness of the film, as shown by the field-dependent nucleation rate determination, $k \cdot N_{\text {diff, }}$ in Figure 4 and the induction times used in fitting the QCM data in Tables 3 and 4. This model can also explain the different Faradaic efficiencies reported in Table 2; the current densities depend on the oxidation of the monomer at the electrode, but the formation of the polymer depends on the radical coupling between two oxidized monomers. If the exchange interactions between the surface and the monomer are attractive, the probability of polymer growth increases. Conversely, if the interactions with the surface are repulsive, the radical is more likely to diffuse away and form soluble oligomers.

\section{CONCLUSIONS}

The present study shows that the electropolymerization reaction of chiral-modified EDOT monomers, 2,3-diphenyl3,4-ethylenedioxythiophene, depends on the spin-polarization of the working electrode and on the chirality of the monomer due to the exchange interactions that arise between the two. The mechanism of the polymer growth is revealed using both electrochemical techniques and QCM measurements, revealing that the exchange interactions effect the electron injection barrier and the nucleation rate at the start of the polymerization. These results are understood within the framework of the CISS effect and illustrate how a similar phenomenon can occur with other chiral monomers. Moreover, these studies demonstrate another instance in which the control of the electron spin, through chirality, can be used to facilitate the kinetics of chemical reactions.

\section{ASSOCIATED CONTENT}

\section{SI Supporting Information}

The Supporting Information is available free of charge at https://pubs.acs.org/doi/10.1021/acs.jpcc.0c06238.

The characterization of the monomers, electrochemical data of the QCM polymerizations, mathematical treatment of the fitting of the QCM data, and electro- chemical and spectroscopic characterization of the polymerized chiral films (PDF)

\section{AUTHOR INFORMATION}

\section{Corresponding Author}

Francesco Tassinari - Department of Chemical and Biological Physics, Weizmann Institute of Science, Rehovot 76100, Israel; (1) orcid.org/0000-0003-4652-9789;

Email: francesco.tassinari@weizmann.ac.il

\section{Authors}

Dana Amsallem - Institute of Chemistry, The Hebrew University of Jerusalem, Jerusalem 91904, Israel

Brian P. Bloom - Department of Chemistry, University of Pittsburgh, Pittsburgh, Pennsylvania 15260, United States; (1) orcid.org/0000-0001-9581-9710

Yiyang Lu - Department of Chemistry, University of Pittsburgh, Pittsburgh, Pennsylvania 15260, United States

Anjan Bedi - Institute of Chemistry, The Hebrew University of Jerusalem, Jerusalem 91904, Israel; 이이.org/0000-00016418-4409

David H. Waldeck - Department of Chemistry, University of Pittsburgh, Pittsburgh, Pennsylvania 15260, United States; () orcid.org/0000-0003-2982-0929

Ori Gidron - Institute of Chemistry, The Hebrew University of Jerusalem, Jerusalem 91904, Israel; 이 orcid.org/0000-00027037-0563

Ron Naaman - Department of Chemical and Biological Physics, Weizmann Institute of Science, Rehovot 76100, Israel; (1) orcid.org/0000-0003-1910-366X

Complete contact information is available at:

https://pubs.acs.org/10.1021/acs.jpcc.0c06238

\section{Notes}

The authors declare no competing financial interest.

\section{ACKNOWLEDGMENTS}

F.T. and R.N. acknowledge partial support from the Minerva Foundation. D.H.W. acknowledges support from the United States National Science Foundation (CBET 1852588).

\section{REFERENCES}

(1) Monzon, L. M. A.; Coey, J. M. D. Magnetic Fields in Electrochemistry: The Lorentz Force. A Mini-Review. Electrochem. Commun. 2014, 42, 38-41.

(2) Monzon, L. M. A.; Coey, J. M. D. Magnetic Fields in Electrochemistry: The Kelvin Force. A Mini-Review. Electrochem. Commun. 2014, 42, 42-45.

(3) Barron, L. D. Can a Magnetic Field Induce Absolute Asymmetric Synthesis? Science 1994, 266 (5190), 1491-1492.

(4) Naaman, R.; Waldeck, D. H. Chiral-Induced Spin Selectivity Effect. J. Phys. Chem. Lett. 2012, 3 (16), 2178-2187.

(5) Ghosh, K. B.; Zhang, W.; Tassinari, F.; Mastai, Y.; Lidor-Shalev, O.; Naaman, R.; Möllers, P.; Nürenberg, D.; Zacharias, H.; Wei, J.; et al. Controlling Chemical Selectivity in Electrocatalysis with Chiral CuO-Coated Electrodes. J. Phys. Chem. C 2019, 123 (5), 3024-3031.

(6) Mtangi, W.; Tassinari, F.; Vankayala, K.; Vargas Jentzsch, A.; Adelizzi, B.; Palmans, A. R. A.; Fontanesi, C.; Meijer, E. W.; Naaman, R. Control of Electrons' Spin Eliminates Hydrogen Peroxide Formation During Water Splitting. J. Am. Chem. Soc. 2017, 139 (7), 2794-2798.

(7) Gazzotti, M.; Arnaboldi, S.; Grecchi, S.; Giovanardi, R.; Cannio, M.; Pasquali, L.; Giacomino, A.; Abollino, O.; Fontanesi, C. Spin- 
Dependent Electrochemistry: Enantio-Selectivity Driven by Chiral-

Induced Spin Selectivity Effect. Electrochim. Acta 2018, 286, 271-278.

(8) Fontanesi, C. Spin-Dependent Electrochemistry: A Novel

Paradigm. Curr. Opin. Electrochem. 2018, 7, 36-41.

(9) Mondal, P. C.; Mtangi, W.; Fontanesi, C. Chiro-Spintronics:

Spin-Dependent Electrochemistry and Water Splitting Using Chiral Molecular Films. Small Methods 2018, 2 (4), 1700313.

(10) Metzger, T. S.; Mishra, S.; Bloom, B. P.; Goren, N.; Neubauer, A.; Shmul, G.; Wei, J.; Yochelis, S.; Tassinari, F.; Fontanesi, C.; et al. The Electron Spin as a Chiral Reagent. Angew. Chem., Int. Ed. 2020, 59 (4), 1653-1658.

(11) Bloom, B. P.; Lu, Y.; Metzger, T.; Yochelis, S.; Paltiel, Y.; Fontanesi, C.; Mishra, S.; Tassinari, F.; Naaman, R.; Waldeck, D. H. Asymmetric Reactions Induced by Electron Spin Polarization. Phys. Chem. Chem. Phys. 2020, In press.

(12) Dong, L.; Zhang, Y.; Duan, X.; Zhu, X.; Sun, H.; Xu, J. Chiral PEDOT-Based Enantioselective Electrode Modification Material for Chiral Electrochemical Sensing: Mechanism and Model of Chiral Recognition. Anal. Chem. 2017, 89 (18), 9695-9702.

(13) Dong, L.; Zhang, L.; Duan, X.; Mo, D.; Xu, J.; Zhu, X. Synthesis and Characterization of Chiral PEDOT Enantiomers Bearing Chiral Moieties in Side Chains: Chiral Recognition and Its Mechanism Using Electrochemical Sensing Technology. RSC Adv. 2016, 6 (14), $11536-11545$.

(14) Dietrich, M.; Heinze, J.; Heywang, G.; Jonas, F. Electrochemical and Spectroscopic Characterization of Polyalkylenedioxythiophenes. J. Electroanal. Chem. 1994, 369 (1-2), 87-92.

(15) Chandrasekhar, P. Conducting Polymers, Fundamentals and Applications; Springer International Publishing: Cham, Switzerland, 2018.

(16) Kołodziejczyk, K.; Miękoś, E.; Zieliński, M.; Jaksender, M.; Szczukocki, D.; Czarny, K.; Krawczyk, B. Influence of Constant Magnetic Field on Electrodeposition of Metals, Alloys, Conductive Polymers, and Organic Reactions. J. Solid State Electrochem. 2018, 22 (6), 1629-1647.

(17) Leventis, N.; Dass, A.; Chandrasekaran, N. Mass Transfer Effects on the Electropolymerization Current Efficiency of 3Methylthiophene in the Magnetic Field. J. Solid State Electrochem. 2007, 11 (6), 727-735.

(18) Tamburri, E.; Orlanducci, S.; Toschi, F.; Terranova, M. L.; Passeri, D. Growth Mechanisms, Morphology, and Electroactivity of PEDOT Layers Produced by Electrochemical Routes in Aqueous Medium. Synth. Met. 2009, 159 (5-6), 406-414.

(19) Grujicic, D.; Pesic, B. Electrodeposition of Copper: The Nucleation Mechanisms. Electrochim. Acta 2002, 47 (18), 29012912.

(20) Michaeli, K.; Beratan, D. N.; Waldeck, D. H.; Naaman, R. Voltage-Induced Long-Range Coherent Electron Transfer through Organic Molecules. Proc. Natl. Acad. Sci. U. S. A. 2019, 116 (13), 5931-5936.

(21) Banerjee-Ghosh, K.; Ben Dor, O.; Tassinari, F.; Capua, E.; Yochelis, S.; Capua, A.; Yang, S.-H.; Parkin, S. S. P.; Sarkar, S.; Kronik, L.; et al. Separation of Enantiomers by Their Enantiospecific Interaction with Achiral Magnetic Substrates. Science 2018, 360 (6395), 1331-1334.

(22) Tassinari, F.; Steidel, J.; Paltiel, S.; Fontanesi, C.; Lahav, M.; Paltiel, Y.; Naaman, R. Enantioseparation by Crystallization Using Magnetic Substrates. Chem. Sci. 2019, 10 (20), 5246-5250.

(23) Naaman, R.; Paltiel, Y.; Waldeck, D. H. Chiral Molecules and the Spin Selectivity Effect. J. Phys. Chem. Lett. 2020, 11 (9), 36603666.

(24) Buchachenko, A. L.; Berdinsky, V. L. Spin Catalysis of Chemical Reactions. J. Phys. Chem. 1996, 100 (47), 18292-18299. 